\title{
Improved high-order bending analysis of double curved sandwich panels subjected to multiple loading condi- tions
}

\section{Abstract}

For the first time, the bending analysis of a double curved sandwich panel was presented which was subjected to point load, uniform distributed load on a patch, and harmonic distributed loads and was based on a new improved higher order sandwich panel theory. Since the cross-sectional warping was accurately modeled by this theory, it did not require any shear correction factor. Also, the present analysis incorporated trapezoidal shape factor (the $1+\mathrm{z} / \mathrm{R}$ terms) of a curved panel element. Geometry was used for the consideration of different radii curvatures of the face sheets, while the core was unique. Unlike most of other reference works, the core can have non-uniform thickness. The governing equations were derived by the principle of minimum potential energy. The effects of types of boundary conditions, types of applied loads, core to panel, and radii curvatures ratios on the bending response were also studied.

\section{Keywords}

Bending, Point load, Uniform distributed load, Doubly curved, Improved higher order theory.

\author{
K. Malekzadeh Fard * \\ M. Livani ${ }^{\mathrm{a}}$ \\ A. veisi and ${ }^{b}$ \\ M. Gholami
}

Department of Structural Analysis and Simulation, Space research institute, MalekAshtar University of Technology, Tehran-Karaj Highway, Post box: 13445-768, Tehran, IRAN.

*Author e-mail: kmalekzadeh@mut.ac.ir

\section{INTRODUCTION}

Sandwich plates are widely used in many engineering applications such as aerospace, automobile, and ship building because of their high strength and stiffness, low weight and durability. These plates are generally consisting of two stiff face sheets and a soft core, which are bonded together. In most cases, the core is consisting of a thick foam polymer or honeycomb material, while thin composite laminates are commonly used as the face sheets. In these structures, the core keeps the face sheets at sufficient distance and transmits the transverse normal and shear loads. Advantages of this construction method are used to obtain the plates with high bending stiffness characteristics and an extremely low weight. To use these structures efficiently, an excellent understanding of their mechanical behavior is needed (Kheirikhah et al. 2011). 


\section{NOTATIONS}

$d V_{t}, d V_{c}, d V_{b}$

$M_{z}^{c}$

$M_{x y}^{i}, M_{y x}^{i}, M_{x x}^{i}, M_{y y}^{i}$

$M_{n x x}^{c}, M_{n x y}^{c}, M_{n y y}^{c}, M_{n y x}^{c}$

$M_{n x z}^{c}, M_{n y z}^{c}, M_{n x z}^{* c}, M_{n y z}^{* c}$

$q_{j}\left(x_{i}, y_{i}\right)$

$Q_{i j}$

$\bar{Q}_{i j}$

$u_{k}, v_{k}, w_{k}$

$u_{c}, v_{c}, w_{c}$

$u_{0}^{i}, v_{0}^{i}, w_{0}^{i}$

$z_{t}, z_{b}, z_{c}$

\section{Greek letters}

$\sigma_{i i}^{j}$

$\sigma_{i i}^{c}$

$\tau_{x y}^{j}, \tau_{x z}^{j}, \tau_{y z}^{j}$

$\tau_{x y}^{c}, \tau_{x z}^{c}, \tau_{y z}^{c}$

$\varepsilon_{0 x x}^{i}, \varepsilon_{0 x y}^{i}, \varepsilon_{0 y y}^{i}, \varepsilon_{0 x z}^{i}, \varepsilon_{0 x z}^{i}$

$\varepsilon_{z z}^{c}, \varepsilon_{x x}^{c}, \varepsilon_{y y}^{c}$

$\gamma_{x z}^{c}, \gamma_{y z}^{c}, \gamma_{x y}^{c}$
Volume element of the top face sheet, the core and the bottom face sheet, respectively

Normal bending moments per unit length of the edge of the core

Bending and shear moments per unit length of the edge $(i=t, b)$

Shear and bending moments per unit length of the edge of the core, $(\mathrm{n}=1,2,3)$

Applied static forces on the top and/or bottom face sheet

Laminate stiffness referred to the principal material coordinates Transformed stiffness

Unknowns of the in-plane displacements of the core $(\mathrm{k}=0,1,2,3)$

Displacement components of the core

Displacement components of the face-sheets, $(i=t, b)$

Normal coordinates in the mid-plane of the top and bottom facesheets and the core

Normal stress in the face sheets, $(\mathrm{i}=\mathrm{x}, \mathrm{y}), \mathrm{j}=(\mathrm{t}, \mathrm{b})$

Normal stress in the core, $(\mathrm{i}=\mathrm{x}, \mathrm{y}, \mathrm{z})$

Shear stresses in the face sheets, $j=(t, b)$

Shear stresses in the core

The mid-plane strain components, $(\mathrm{i}=\mathrm{t}, \mathrm{b})$

Normal strain components of the core

Shear strain components of the core 
In order to investigate free vibration and bending analyses of sandwich structures, the higher order sandwich panel theory was developed by Frostig et al. (1995, 1996, 2004) who considered two types of computational models for expressing the governing equations of the core. The second model assumed a polynomial description of the displacement fields in the core that was based on the displacement fields of the first model. Their theory does not impose any restriction on the deformation distribution through the thickness of the core. Bozhevolnaya and Frostig (1997) studied the static analysis of open single curved sandwich panels and uses high order theory to demonstrate the influence of the geometrical nonlinearity on the overall and local behaviors of the sandwich panel. The postbuckling analysis of geometrically imperfect anisotropic sandwich doubly-curved panels under compressive edge loads and a lateral pressure was done by Hause et al. (1998) who assumed the core and the face sheets to be incompressible in the transverse normal direction. Frostig (1999) using higher order sandwich panel theory studied the bending of an open single curved panel with arbitrary boundary conditions. He modeled effects of the flexibility of the core of the sandwich panel. Petras and Sutcliffe (1999) applied the higher order sandwich beam theory and studied the bending of sandwich beams. In their theory, the shear stress in thickness directions was assumed to be uniformly distributed; but, a second order function was considered for the vertical displacement of the core. The improved higher order sandwich plate theory, applying the first order shear deformation theory for the face sheets, was introduced by Malekzadeh et al. (2005a). Zenkour (2005a, b) presented the comprehensive analysis of FG sandwich plates. The face sheets were assumed to be isotropic and two-constituentional material distribution through the thickness was assumed to vary according to the power law distribution. Bending, buckling, and free vibration of simply supported FG ceramic-metal sandwich plates were also investigated. The dynamic buckling and postbuckling analyses of doubly curved sandwich panels were investigated by Hohe et al. (2006) in which the standard Kirchhoff-Love hypothesis for the face sheets and a first/second order power series expansion for the core was used. They ignored the transverse shear strains of the core layer. Garg et al.. (2006) investigated the free vibration analysis of simply supported doubly curved sandwich shells and their formulation included the Sander's theory and they assumed the parabolic distribution of transverse shear strains through the shell thickness. Experimental and analytical investigations of the bending and free vibration analyses of layered FG beams were carried out by Kapuria et al. (2008) who demonstrated the capability of the zigzag theory in modeling such beams. Rahmani et al. (2009) applied a higher order sandwich panel theory to study the free vibration analysis of an open single curved composite sandwich panel with a flexible core. They used the classical shell theory and an elasticity theory for the face sheets and the core, respectively. Carrera and Brischetto (2009) used classical plate, zig-zag, and high order theories and studied the bending of open single curved composite sandwich panels. Using these theories, they studied the effects of geometrical parameters (length to thickness ratio) and mechanical parameters (face sheets to core stiffness ratio) on the bending response and concluded that the higher order theories could increase the accuracy of the obtained results. Cetkovic and Vuksanovic (2009) investigated the global and local responses of sandwich structures based on Reddy's layerwise theory. Also, they used finite element method and investigated the free vibration, bending, and buckling analyses of sandwich structures. Deformation and failure modes of open single curved sandwich panels with 
two aluminium face sheets and an aluminium foam core subjected to the air blast loadings were experimentally investigated by Shen et al. (2010). All the four edges of the panels were fully clamped. Biglari and Jafari (2010) presented a complex three layer theory for the free vibration and bending analysis of open single curved sandwich structures. In their model, Donell's theory was used for the face sheets. Zhen and Wanji (2010) applied a $\mathrm{C}^{0}$ type higher order equivalent single layer theory to study the bending analysis of laminated composite and sandwich plates subjected to the thermal and mechanical loads. The continuity conditions of transverse shear stresses at interfaces and the conditions of zero transverse shear stresses on the upper and lower surfaces were also considered. The bending analysis of laminated composite plates under bisinusoidal loading was done by Stürzenbecher and Hofstetter (2011). In their theory, by using an equivalent single layer plate theory, the transverse shear strains jumped at the layer interfaces, but transverse shear stresses were continuous and the normal stress was ignored. Kheirikhah et al. (2011) investigated the bending analysis of sandwich panels with flexible cores. He et al. (2012) performed the bending analysis of sandwich panels with different core geometries including corrugated, honeycomb, and X cores while neglecting transverse shear strains of the face sheets. Classical and first order shear deformation theories were employed for the face sheets and the core, respectively. Stacking sequence for composite panels under slamming impact loads was optimized by Khedmati et al. (2013). who wrote a special code in MATLAB based on a genetic algorithm method and coupled it with ANSYS in order to calculate the central deflection of the composite panel. Neto et al. (2014) presented a new metamodel for the reinforced panels made of aluminum alloy under compressive loads based on the synthesis of four stability criteria: section crippling, web buckling, flange buckling, and column collapse.

The literature survey demonstrated that most of the studies have been performed on the bending analysis of flat composite sandwich panels and the free vibration and buckling analyses of double curved sandwich panels under simple loadings and no research is available on the bending analysis of double curved sandwich panels. In this paper, sandwich structures were subjected to multiple loading conditions including point load, uniform distributed load on a patch, harmonic, and uniform distributed loads which were imposed on the top and/or bottom face sheets of the sandwich structure. Geometries were used in the present work for the consideration of different radii curvatures of the face sheets and the core was unique. In this paper, unlike most of other works, in which the core is assumed to be uniform throughout the entire panel, the core was supposed to have nonuniform thickness. As a result, this study was able to analyse a wide range of sandwich panels.

In this paper, by using an improved higher order sandwich panel theory (Malekzadeh et al. 2005a, b) and the second computational Frostig's model (2004), the static bending analysis of double curved composite sandwich panels was investigated. Also, the in-plane hoop stresses of the core were considered. In this study, the analytical solution of the displacement field of the core in terms of the polynomials with unknown coefficients was presented according to the second computational Frostig's model (2004). Moreover, the formulation included accurate stressresultant equations for the composite sandwich structures, where $\left(1+z_{c} / R_{x c}\right)$ and $\left(1+z_{c} / R_{y c}\right)$ terms were imported in the stress-resultant equations and exactly integrated. These coefficients could be very important in the bending analysis of double curved composite sandwich 
structures. Simply supported and full clamped boundary conditions were considered in this paper. In order to assure the accuracy of the present formulations, the convergence of the results was examined in detail. The numerical results of the analysis were compared with the available experimental and theoretical results in the literature or those presented in FE model by ABAQUS code. Finally, the effects of various parameters including radii curvatures, core to panel thickness ratio, composite layup sequence, types of applied loads, and types of boundary conditions on the bending analysis were studied.

\section{THEORETICAL FORMULATION}

\subsection{Basic Assumptions}

Consider a doubly curved composite sandwich panel which is composed of two composite laminated face sheets. The thickness of the top face sheet, bottom face sheet and the core is $h_{t}, h_{b}$ and $h_{c}$, respectively. According to Figure 1, the length, width and total thickness of the panel are a, b and $\mathrm{h}$, respectively. The orthogonal curvilinear coordinates $\left(\mathrm{x}_{\mathrm{i}}, \mathrm{y}_{\mathrm{i}}, \mathrm{z}_{\mathrm{i}}, \mathrm{i}=\mathrm{t}, \mathrm{b}, \mathrm{c}\right)$ are also shown in

Figure 1 in which indices $t$ and $b$ refer to the top and bottom face sheets of the panel, respectively. The curvature radii of the top face sheet, bottom face sheet and the core in $\mathrm{x}-\mathrm{z}$ and $\mathrm{y}-\mathrm{z}$ planes are $R_{\mathrm{tx}}, \mathrm{R}_{\mathrm{bx}}, \mathrm{R}_{\mathrm{cx}}$, and $\mathrm{R}_{\mathrm{ty}}, \mathrm{R}_{\mathrm{by}}, \mathrm{R}_{\mathrm{cy}}$, respectively. The assumption used in the present analysis was the small deformation of linearly elastic materials.

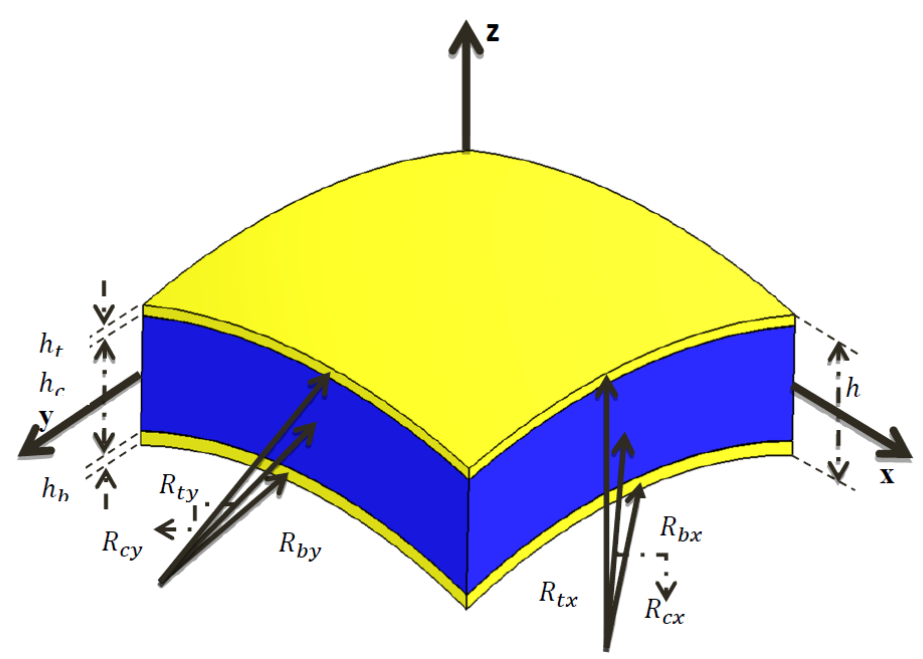

Figure 1 A double curved sandwich panel with laminated face sheets and the orthogonal curvilinear coordinates.

\subsection{Kinematic relations}

Based on the first shear deformation theory, the displacements $\mathrm{u}, \mathrm{v}$ and $\mathrm{w}$ of the face sheets in the $\mathrm{x}, \mathrm{y}$ (longitudinal), and $\mathrm{z}$ (thickness) directions with small linear displacements are expressed by following relations (Reddy, 2003): 


$$
\begin{aligned}
& u_{i}(x, z, y, t)=u_{0}^{i}(x, y, t)+z_{i} \psi_{x}^{i}(x, y, t) \\
& v_{i}(x, z, y, t)=v_{0}^{i}(x, y, t)+z_{i} \psi_{y}^{i}(x, y, t) \quad ; \quad(i=t, b) \\
& w_{i}(x, z, y, t)=w_{0}^{i}(x, y, t)
\end{aligned}
$$

where $z_{i}$ is the vertical coordinate of each face-sheet $(\mathrm{i}=\mathrm{t}, \mathrm{b})$ and is measured upward from the mid-plane of each face-sheet. The kinematic equations for the strains of the face sheets are as follows:

$$
\begin{array}{ll}
\varepsilon_{x x}^{i}=\varepsilon_{0 x x}^{i}+z_{i} \kappa_{x x}^{i}, & \varepsilon_{y y}^{i}=\varepsilon_{0 y y}^{i}+z_{i} \kappa_{y y}^{i} \varepsilon_{z z}^{i}=0 \\
\gamma_{x y}^{i}=2 \varepsilon_{x y}^{i}=\varepsilon_{0 x y}^{i}+z_{i} \kappa_{x y}^{i}, \gamma_{x z}^{i}=2 \varepsilon_{x z}^{i}=\varepsilon_{0 x z}^{i}, & \gamma_{y z}^{i}=2 \varepsilon_{y z}^{i}=\varepsilon_{0 y z}^{i},
\end{array} \quad ; \quad(i=t, b)
$$

where

$$
\begin{array}{ll}
\varepsilon_{0 x x}^{i}=\frac{\partial u_{0}^{i}}{\partial x}+\frac{w_{0}^{i}}{R_{x i}}, \quad \varepsilon_{0 y y}^{i}=\frac{\partial v_{0}^{i}}{\partial y}+\frac{w_{0}^{i}}{R_{y i}}, & \\
\varepsilon_{0 x y}^{i}=\frac{\partial v_{0}^{i}}{\partial x}+\frac{\partial u_{0}^{i}}{\partial y}, \quad \varepsilon_{0 x z}^{i}=\frac{\partial w_{0}^{i}}{\partial x}+\psi_{x}^{i}-\frac{u_{0}^{i}}{R_{x i}}, \quad \varepsilon_{0 y z}^{i}=\frac{\partial w_{0}^{i}}{\partial y}+\psi_{y}^{i}-\frac{v_{0}^{i}}{R_{y i}} \\
\kappa_{x x}^{i}=\frac{\partial \psi_{x}^{i}}{\partial x}, \quad \kappa_{y y}^{i}=\frac{\partial \psi_{y}^{i}}{\partial y}, \quad \kappa_{x y}^{i}=\frac{\partial \psi_{y}^{i}}{\partial x}+\frac{\partial \psi_{x}^{i}}{\partial y}
\end{array}
$$

The displacement fields are based on the second Frostig's model (2004) for the thick core and a cubic pattern for in-plane displacements and a quadratic one for vertical ones are taken as follows:

$$
\left\{\begin{array}{l}
u_{c}(x, y, z, t)=\left(1+\frac{z}{R_{x c}}\right) u_{0}^{c}(x, y, t)+z_{c} u_{1}^{c}(x, y, t)+z_{c}^{2} u_{2}^{c}(x, y, t)+z_{c}^{3} u_{3}^{c}(x, y, t) \\
v_{c}(x, y, z, t)=\left(1+\frac{z}{R_{y c}}\right) v_{0}^{c}(x, y, t)+z_{c} v_{1}^{c}(x, y, t)+z_{c}^{2} v_{2}^{c}(x, y, t)+z_{c}^{3} v_{3}^{c}(x, y, t) \\
w_{c}(x, y, z, t)=w_{0}^{c}(x, y, t)+z_{c} w_{1}^{c}(x, y, t)+z_{c}^{2} w_{2}^{c}(x, y, t)
\end{array}\right.
$$

The kinematic relations of the core for doubly curved sandwich panels based on small deformations are: 


$$
\begin{aligned}
\varepsilon_{x x}^{c} & =\frac{1}{\left(1+z / R_{x c}\right)}\left(\frac{\partial u_{c}}{\partial x}+\frac{w_{c}}{R_{x c}}\right) \\
c & \left.\frac{1}{\varepsilon_{y y}}=\frac{\partial v_{c}}{\left(1+z / R_{y c}\right)}+\frac{w_{c}}{R_{y c}}\right) \\
c & c_{c}=\frac{1}{\left(1+z / R_{x c}\right)} \frac{\partial v_{c}}{\partial x}+\frac{1}{\left(1+z / R_{y c}\right)} \frac{\partial u_{c}}{\partial y} \\
\gamma_{x y} & =2 \varepsilon_{x y}=\frac{1}{c}\left(\frac{\partial w_{c}}{\partial x}-\frac{u_{c}}{R_{x c}}\right)+\frac{\partial u_{c}}{\partial z} \\
\gamma_{x z} & \left.=2 \varepsilon_{x z}=\frac{1}{\left(1+z / R_{x c}\right.}\right)\left(\frac{\partial w_{c}}{\partial y}-\frac{v_{c}}{R_{y c}}\right)+\frac{\partial v_{c}}{\partial z} \\
\gamma_{y z} & =2 \varepsilon_{y z}=\frac{1}{\left(1+z / R_{y c}\right)}
\end{aligned}
$$

\subsection{Compatibility conditions}

The compatibility conditions are presented by assuming perfect bonding between the core and the face-sheets (Kheirikhah et al. 2011):

$$
\left\{\begin{array} { l } 
{ u _ { c } ( z = z _ { c i } ) = u _ { 0 } ^ { i } + \frac { 1 } { 2 } ( - 1 ) ^ { k } h _ { i } \psi _ { x } ^ { i } } \\
{ v _ { c } ( z = z _ { c i } ) = v _ { 0 } ^ { i } + \frac { 1 } { 2 } ( - 1 ) ^ { k } h _ { i } \psi _ { y } ^ { i } } \\
{ w _ { c } ( z = z _ { c i } ) = w _ { 0 } ^ { i } }
\end{array} \quad \left\{\begin{array}{l}
\text { For } i=t \rightarrow\left(k=1 ; z_{c t}=\frac{h_{c}}{2}\right) \\
\text { For } i=b \rightarrow\left(k=0 ; z_{c b}=-\frac{h_{c}}{2}\right)
\end{array}\right.\right.
$$

By using the displacement fields of the core (Equation (4) and Equation (6)) and also by simplifying, the compatibility conditions can be written as follow:

$$
\left\{\begin{array}{l}
u_{2}^{c}=\frac{2\left(u_{0}^{t}+u_{0}^{b}\right)-h_{t} \psi_{x}^{t}+h_{b} \psi_{x}^{b}-4 u_{0}^{c}}{h_{c}^{2}} \\
u_{3}^{c}=\frac{4\left(u_{0}^{t}-u_{0}^{b}\right)-2\left(h_{t} \psi_{x}^{t}+h_{b} \psi_{x}^{b}\right)-4 h_{c} u_{1}^{c}-4 h_{c} u_{0}^{c} / R_{x c}}{h_{c}^{3}} \\
v_{2}^{c}=\frac{2\left(v_{0}^{t}+v_{0}^{b}\right)-h_{t} \psi_{y}^{t}+h_{b} \psi_{y}^{b}-4 v_{0}^{c}}{h_{c}^{2}} \\
v_{3}^{c}=\frac{4\left(v_{0}^{t}-v_{0}^{b}\right)-2\left(h_{t} \psi_{y}^{t}+h_{b} \psi_{y}^{b}\right)-4 h_{c} v_{1}^{c}-4 h_{c} v_{0}^{c} / R_{y c}}{h_{c}^{3}} u_{0}^{c}, u_{1}^{c}, v_{0}^{c}, v_{1}^{c} \text { and } w_{0}^{c} \\
w_{1}^{c}=\frac{\left(w_{0}^{t}-w_{0}^{b}\right)}{h_{c}} \\
w_{2}^{c}=\frac{2\left(w_{0}^{t}+w_{0}^{b}\right)-4 w_{0}^{c}}{h_{c}^{2}}
\end{array}\right.
$$


It can be seen from Equation (7) that the number of unknowns in the core is reduced to five. These unknowns are $u_{0}^{c}, u_{1}^{c}, v_{0}^{c}, v_{1}^{c}$ and $w_{0}^{c}$. Therefore in a general form, the number of unknowns for a doubly curved composite sandwich panel is fifteen as shown below:

$$
\left\{u_{0}^{t}, v_{0}^{t}, w_{0}^{t}, \psi_{x}^{t}, \psi_{y}^{t}, u_{0}^{b}, v_{0}^{b}, w_{0}^{b}, \psi_{x}^{b}, \psi_{y}^{b}, u_{0}^{c}, u_{1}^{c}, v_{0}^{c}, v_{1}^{c}, w_{0}^{c}\right\}
$$

\subsection{Governing equations}

The equilibrium equation for the face sheets and the core are derived by the principle of the minimum potential energy:

$$
\delta \Pi=\delta U+\delta W_{e x t}=0
$$

where $\delta U$ and $\delta W_{\text {ext }}$ denote the variation of strain energy and that of potential energy due to the applied loads, respectively. Also $\delta$ denotes the variation operator.

The first variation of the internal potential energy for a doubly curved sandwich panel that includes the face sheets and the core is:

$$
\begin{aligned}
\delta U= & \sum_{i=t, b}\left(\int_{V_{i}}\left(\sigma_{x x}^{i} \delta \varepsilon_{x x}^{i}+\sigma_{y y}^{i} \delta \varepsilon_{y y}^{i}+\tau_{x y}^{i} \delta \gamma_{x y}^{i}+\tau_{x z}^{t} \delta \gamma_{x z}^{i}+\tau_{y z}^{i} \delta \gamma_{y z}^{i}\right) d V_{i}\right) \\
& +\int_{V_{c}}\left(\sigma_{x x}^{c} \delta \varepsilon_{x x}^{c}+\sigma_{y y}^{c} \delta \varepsilon_{y y}^{c}+\sigma_{z z}^{c} \delta \varepsilon_{z z}^{c}+\tau_{x y}^{c} \delta \gamma_{x y}^{c}+\tau_{x z}^{c} \delta \gamma_{x z}^{c}+\tau_{y z}^{c} \delta \gamma_{y z}^{c}\right) d V_{c} \\
d V_{c} & =d A_{c} d z_{c}=\left(1+\frac{z_{c}}{R_{x c}}\right)\left(1+\frac{z_{c}}{R_{y c}}\right) d x_{c} d y_{c} d z_{c}, d V_{i}=d A_{i} d z_{i}=d x_{i} d y_{i} d z_{i},(i=t, b) .
\end{aligned}
$$

The variation of the external work is the summation of the applied loads on the top and bottom face sheets and on edges:

$$
\delta W_{e x t}=\int_{A}\left(-\left(1+\frac{h_{t}}{2 R_{x t}}\right)\left(1+\frac{h_{t}}{2 R_{y t}}\right) q_{t} \delta w_{0}^{t}+\left(1-\frac{h_{b}}{2 R_{x b}}\right)\left(1-\frac{h_{b}}{2 R_{y b}}\right) q_{b} \delta w_{0}^{b}\right) d x d y
$$

By using the principle of minimum potential energy (Equations (8)-(10)) and kinematic relations (Equations (1)-(7), the governing in the system of orthogonal curvilinear coordinates $(\mathrm{x}, \mathrm{y}, \mathrm{z})$ can be obtained as:

$$
\begin{gathered}
\delta u_{0}^{t}: \quad N_{x x, x}^{t}+N_{x y, y}^{t}+C_{0} M_{x y, y}^{t}+\frac{Q_{x z}^{t}}{R_{t x}}+\frac{2}{h_{c}^{2}} M_{2 x x, x}^{c}+\frac{4}{h_{c}^{3}} M_{3 x x, x}^{c}+\frac{2}{h_{c}^{2}} M_{2 y x, y}^{c}+\frac{4}{h_{c}^{3}} M_{3 y x, y}^{c}+ \\
\frac{2}{R_{t x} h_{c}^{2}} M_{2 x z}^{c}+\frac{4}{R_{t x} h_{c}^{3}} M_{3 x z}^{c}-\frac{4}{h_{c}^{2}} M_{1 x z}^{* c}-\frac{12}{h_{c}^{3}} M_{2 x z}^{* c}=0
\end{gathered}
$$


$\delta u_{0}^{b}: \quad N_{x x, x}^{b}+N_{x y, y}^{b}+C_{0} M_{x y, y}^{b}+\frac{Q_{x z}^{b}}{R_{b x}}+\frac{2}{h_{c}^{2}} M_{2 x x, x}^{c}-\frac{4}{h_{c}^{3}} M_{3 x x, x}^{c}+\frac{2}{h_{c}^{2}} M_{2 y x, y}^{c}-\frac{4}{h_{c}^{3}} M_{3 y x, y}^{c}+$

$$
\frac{2}{R_{b x} h_{c}^{2}} M_{2 x z}^{c}-\frac{4}{R_{b x} h_{c}^{3}} M_{3 x z}^{c}-\frac{4}{h_{c}^{2}} M_{1 x z}^{* c}+\frac{12}{h_{c}^{3}} M_{2 x z}^{*_{c}}=0
$$

$\delta v_{0}^{t}: \quad N_{x y, x}^{t}+N_{y y, y}^{t}+\frac{Q_{y z}^{t}}{R_{t y}}+\frac{2}{h_{c}^{2}} M_{2 y y, y}^{c}+\frac{4}{h_{c}^{3}} M_{3 y y, y}^{c}+\frac{2}{h_{c}^{2}} M_{2 x y, x}^{c}+\frac{4}{h_{c}^{3}} M_{3 x y, x}^{c}+\frac{2}{R_{c y} h_{c}^{2}} M_{2 y z}^{c}+$

$$
\frac{4}{R_{c y} h_{c}^{3}} M_{3 y z}^{c}-\frac{4}{h_{c}^{2}} M_{1 y z}^{*_{c}}-\frac{12}{h_{c}^{3}} M_{2 y z}^{*_{c}}=0
$$

$\delta v_{0}^{b}: \quad N_{x y, x}^{b}+N_{y y, y}^{b}+\frac{Q_{y z}^{b}}{R_{b y}}+\frac{2}{h_{c}^{2}} M_{2 y y, y}^{c}-\frac{4}{h_{c}^{3}} M_{3 y y, y}^{c}+\frac{2}{h_{c}^{2}} M_{2 x y, x}^{c}-\frac{4}{h_{c}^{3}} M_{3 x y, x}^{c}+\frac{2}{R_{c y} h_{c}^{2}} M_{2 y z}^{c}-$

$$
\frac{4}{R_{c y} h_{c}^{3}} M_{3 y z}^{c}-\frac{4}{h_{c}^{2}} M_{1 y z}^{*_{c}}+\frac{12}{h_{c}^{3}} M_{2 y z}^{*_{c}}=0
$$

$\delta w_{0}^{t}: \quad Q_{x z, x}^{t}+Q_{y z, y}^{t}-\left(\frac{N_{x x}^{t}}{R_{t x}}+\frac{N_{y y}^{t}}{R_{t y}}\right)-\frac{R_{z}^{c}}{h_{c}}-\frac{1}{R_{c x} h_{c}} M_{1 x x}^{c}-\frac{4}{h_{c}^{2}} M_{z}^{c}-\frac{2}{R_{c x} h_{c}^{2}} M_{2 x x}^{c}-\frac{1}{R_{c y} h_{c}} M_{1 y y}^{c}$ $-\frac{2}{R_{c y} h_{c}^{2}} M_{2 y y}^{c}+\frac{1}{h_{c}} M_{1 x z, x}^{c}+\frac{2}{h_{c}^{2}} M_{2 x z, x}^{c}+\frac{1}{h_{c}} M_{1 y z, y}^{c}+\frac{2}{h_{c}^{2}} M_{2 y z, y}^{c}-\left(1+\frac{h_{t}}{2 R_{t x}}\right)\left(1+\frac{h_{t}}{2 R_{t y}}\right) q_{t}=0$

$\delta w_{0}^{b}: \quad Q_{x z, x}^{b}+Q_{y z, y}^{b}-\left(\frac{N_{x x}^{b}}{R_{b x}}+\frac{N_{y y}^{b}}{R_{b y}}\right)+\frac{R_{z}^{c}}{h_{c}}+\frac{1}{R_{c x} h_{c}} M_{1 x x}^{c}-\frac{4}{h_{c}^{2}} M_{z}^{c}-\frac{2}{R_{c x} h_{c}^{2}} M_{2 x x}^{c}+\frac{1}{R_{c y} h_{c}} M_{1 y y}^{c}$ $-\frac{2}{R_{c y} h_{c}^{2}} M_{2 y y}^{c}-\frac{1}{h_{c}} M_{1 x z, x}^{c}+\frac{2}{h_{c}^{2}} M_{2 x z, x}^{c}-\frac{1}{h_{c}} M_{1 y z, y}^{c}+\frac{2}{h_{c}^{2}} M_{2 y z, y}^{c}+\left(1-\frac{h_{b}}{2 R_{b x}}\right)\left(1-\frac{h_{b}}{2 R_{b y}}\right) q_{b}=0$

$\delta \psi_{x}^{t}: \quad M_{x x, x}^{t}+M_{x y, y}^{t}-Q_{x z}^{t}-\frac{h_{t}}{h_{c}^{2}} M_{2 x x, x}^{c}-\frac{2 h_{t}}{h_{c}^{3}} M_{3 x x, x}^{c}-\frac{h_{t}}{h_{c}^{2}} M_{2 y x, y}^{c}-\frac{2 h_{t}}{h_{c}^{3}} M_{3 y x, y}^{c}-\frac{h_{t}}{R_{c x} h_{c}^{2}} M_{2 x z}^{c}$

$$
-\frac{2 h_{t}}{R_{c x} h_{c}^{2}} M_{3 x z}^{c}+\frac{2 h_{t}}{h_{c}^{2}} M_{1 x z}^{* c}+\frac{6 h_{t}}{h_{c}^{3}} M_{2 x z}^{*_{c}}=0
$$

$\delta \psi_{x}^{b}: \quad M_{x x, x}^{b}+M_{x y, y}^{b}-Q_{x z}^{b}+\frac{h_{b}}{h_{c}^{2}} M_{2 x x, x}^{c}-\frac{2 h_{b}}{h_{c}^{3}} M_{3 x x, x}^{c}+\frac{h_{b}}{h_{c}^{2}} M_{2 y x, y}^{c}-\frac{2 h_{b}}{h_{c}^{3}} M_{3 y x, y}^{c}+\frac{h_{b}}{R_{c x} h_{c}^{2}} M_{2 x z}^{c}$

$$
-\frac{2 h_{b}}{R_{c x} h_{c}^{2}} M_{3 x z}^{c}-\frac{2 h_{b}}{h_{c}^{2}} M_{1 x z}^{* c}+\frac{6 h_{b}}{h_{c}^{3}} M_{2 x z}^{*_{c}}=0
$$




$$
\begin{gathered}
\delta \psi_{y}^{t}: M_{x y, x}^{t}+M_{y y, y}^{t}-Q_{y z}^{t}-\frac{h_{t}}{h_{c}^{2}} M_{2 y y, y}^{c}-\frac{2 h_{t}}{h_{c}^{3}} M_{3 y y, y}^{c}-\frac{h_{t}}{h_{c}^{2}} M_{2 x y, x}^{c}-\frac{2 h_{t}}{h_{c}^{3}} M_{3 x y, x}^{c}-\frac{h_{t}}{R_{c x} h_{c}^{2}} M_{2 y z}^{c}- \\
\frac{2 h_{t}}{R_{c x} h_{c}^{2}} M_{3 y z}^{c}+\frac{2 h_{t}}{h_{c}^{2}} M_{1 y z}^{*}+\frac{6 h_{t}}{h_{c}^{3}} M_{2 y z}^{*}=0
\end{gathered}
$$

$\delta \psi_{y}^{b}: \quad M_{x y, x}^{b}+M_{y y, y}^{b}-Q_{y z}^{b}+\frac{h_{b}}{h_{c}^{2}} M_{2 y y, y}^{c}-\frac{2 h_{b}}{h_{c}^{3}} M_{3 y y, y}^{c}+\frac{h_{b}}{h_{c}^{2}} M_{2 x y, x}^{c}-\frac{2 h_{b}}{h_{c}^{3}} M_{3 x y, x}^{c}+\frac{h_{b}}{R_{c y} h_{c}^{2}} M_{2 y z}^{c}-$

$$
\frac{2 h_{b}}{R_{c y} h_{c}^{2}} M_{3 y z}^{c}-\frac{2 h_{b}}{h_{c}^{2}} M_{1 y z}^{*}+\frac{6 h_{b}}{h_{c}^{3}} M_{2 y z}^{* c}=0
$$

$\delta u_{0}^{c}: \quad N_{x x, x}^{c}+N_{y x, y}^{c}-\frac{4}{h_{c}^{2}} M_{2 x x, x}^{c}-\frac{4}{R_{c x} h_{c}^{2}} M_{3 x x, x}^{c}+\frac{1}{R_{c x}} M_{1 y x, y}^{c}-\frac{4}{h_{c}^{2}} M_{2 y x, y}^{c}-\frac{4}{R_{c x} h_{c}^{2}} M_{3 y x, y}^{c}+$

$$
\frac{1}{R_{c x}} N_{x z}^{c}-\frac{4}{R_{c x} h_{c}^{2}} M_{2 x z}^{c}-\frac{4}{R_{c x}^{2} h_{c}^{2}} M_{3 x z}^{c}+\frac{8}{h_{c}^{2}} M_{1 x z}^{*}+\frac{12}{R_{c x} h_{c}^{2}} M_{2 x z}^{* c}-\frac{1}{R_{c x}} N_{x z}^{* c}=0
$$

$\delta u_{1}^{c}: M_{1 x x, x}^{c}-N_{x z}^{* c}-\frac{4}{h_{c}^{2}} M_{3 x x, x}^{c}+M_{1 y x, y}^{c}-\frac{4}{h_{c}^{2}} M_{3 y x, y}^{c}+\frac{1}{R_{c x}} M_{1 x z}^{c}-\frac{4}{R_{c x} h_{c}^{2}} M_{3 x z}^{c}+\frac{12}{h_{c}^{2}} M_{2 x z}^{*_{c}}=0$

$$
\begin{gathered}
\delta v_{0}^{c}: \quad N_{y y, y}^{c}+N_{x y, x}^{c}-\frac{4}{h_{c}^{2}} M_{2 y y, y}^{c}-\frac{4}{R_{c y} h_{c}^{2}} M_{3 y y, y}^{c}+\frac{1}{R_{c y}} M_{1 x y, x}^{c}-\frac{4}{h_{c}^{2}} M_{2 x y, x}^{c}-\frac{4}{R_{c y} h_{c}^{2}} M_{3 x y, x}^{c}+ \\
\frac{1}{R_{c y}} N_{y z}^{c}-\frac{4}{R_{c y} h_{c}^{2}} M_{2 y z}^{c}-\frac{4}{R_{c y}^{2} h_{c}^{2}} M_{3 y z}^{c}+\frac{8}{h_{c}^{2}} M_{1 y z}^{*}+\frac{12}{R_{c y} h_{c}^{2}} M_{2 y z}^{*}-\frac{1}{R_{c y}} N_{y z}^{*}=0
\end{gathered}
$$

$\delta v_{1}^{c}: M_{1 y y, y}^{c}-N_{y z}^{*_{c}}-\frac{4}{h_{c}^{2}} M_{3 y y, y}^{c}+M_{1 x y, x}^{c}-\frac{4}{h_{c}^{2}} M_{3 x y, x}^{c}+\frac{1}{R_{c y}} M_{1 y z}^{c}-\frac{4}{R_{c y} h_{c}^{2}} M_{3 y z}^{c}+\frac{12}{h_{c}^{2}} M_{2 y z}^{*}=0$

$$
\begin{gathered}
\delta w_{o}^{c}: \quad N_{x z, x}^{c}+N_{y z, y}^{c}+\frac{8}{h_{c}^{2}} M_{z}^{c}-\frac{1}{R_{x c}} N_{x x}^{c}+\frac{4}{R_{x c} h_{c}^{2}} M_{2 x x}^{c}-\frac{1}{R_{y c}} N_{y y}^{c}+\frac{4}{R_{y c} h_{c}^{2}} M_{2 y y}^{c}-\frac{4}{h_{c}^{2}} M_{2 x z, x}^{c} \\
-\frac{4}{h_{c}^{2}} M_{2 y z, y}^{c}=0
\end{gathered}
$$

where the stress resultants per unit length for the core can be defined as follow: 


$$
\begin{aligned}
& \left\{\begin{array}{c}
N_{x x}^{c} \\
N_{y y}^{c} \\
N_{x y}^{c} \\
N_{y x}^{c}
\end{array}\right\}=\int_{-h_{c} / 2}^{h_{c} / 2}\left\{\begin{array}{c}
\sigma_{x x}^{c}\left(1+\frac{z_{c}}{R_{y c}}\right) \\
\sigma_{y y}^{c}\left(1+\frac{z_{c}}{R_{x c}}\right) \\
\sigma_{x y}^{c}\left(1+\frac{z_{c}}{R_{y c}}\right) \\
\sigma_{x y}^{c}\left(1+\frac{z_{c}}{R_{x c}}\right)
\end{array}\right\} d z_{c}, \quad\left\{\begin{array}{c}
M_{n x x}^{c} \\
M_{n y y}^{c} \\
M_{n x y}^{c} \\
M_{n y x}^{c}
\end{array}\right\}=\int_{-h_{c} / 2}^{h_{c} / 2} z_{c}^{n}\left\{\begin{array}{l}
\sigma_{x x}^{c}\left(1+\frac{z_{c}}{R_{y c}}\right) \\
\sigma_{y y}^{c}\left(1+\frac{z_{c}}{R_{x c}}\right) \\
\sigma_{x y}^{c}\left(1+\frac{z_{c}}{R_{y c}}\right) \\
\sigma_{x y}^{c}\left(1+\frac{z_{c}}{R_{x c}}\right)
\end{array}\right\} d z_{c}, \\
& \left\{\begin{array}{l}
N_{x z}^{c} \\
N_{y z}^{c} \\
M_{n x z}^{c} \\
M_{n y z}^{c}
\end{array}\right\}=\int_{-h_{c} / 2}^{h_{c} / 2}\left\{\begin{array}{c}
\sigma_{x z}^{c}\left(1+\frac{z_{c}}{R_{y c}}\right) \\
\sigma_{y z}^{c}\left(1+\frac{z_{c}}{R_{x c}}\right) \\
z_{c}^{n} \sigma_{x z}^{c}\left(1+\frac{z_{c}}{R_{y c}}\right) \\
z_{c}^{n} \sigma_{y z}^{c}\left(1+\frac{z_{c}}{R_{x c}}\right)
\end{array}\right\} d z_{c}, \quad\left\{\begin{array}{l}
N_{x z}^{* c} \\
N_{y z}^{* c} \\
M_{n x z}^{* c} \\
M_{n y z}^{* c}
\end{array}\right\}=\int_{-h_{c} / 2}^{h_{c} / 2}\left\{\begin{array}{l}
\sigma_{x z}^{c} \\
\sigma_{y z}^{c} \\
z_{c}^{n} \sigma_{x z}^{c} \\
z_{c}^{n} \sigma_{y z}^{c}
\end{array}\right\}\left(1+\frac{z_{c}}{R_{x c}}\right)\left(1+\frac{z_{c}}{R_{y c}}\right) d z_{c}, \\
& \left\{R_{z}^{c}, M_{z}^{c}\right\}=\int_{-h_{c} / 2}^{h_{c} / 2}\left(1, z_{c}\right) \sigma_{z z}^{c}\left(1+\frac{z_{c}}{R_{x c}}\right)\left(1+\frac{z_{c}}{R_{y c}}\right) d z_{c}, \quad\left\{\begin{array}{c}
N_{x x}^{i} \\
N_{y y}^{i} \\
N_{x y}^{i} \\
N_{y x}^{i}
\end{array}\right\}=\int_{-h_{i} / 2}^{h_{i} / 2}\left\{\begin{array}{c}
\sigma_{x x}^{i} \\
\sigma_{y y}^{i} \\
\sigma_{x y}^{i} \\
\sigma_{x y}^{i}
\end{array}\right\} d z_{i}, \\
& \left\{\begin{array}{c}
M_{x x}^{i} \\
M_{y y}^{i} \\
M_{x y}^{i} \\
M_{y x}^{i}
\end{array}\right\}=\int_{-h_{i} / 2}^{h_{i} / 2} z_{i}\left\{\begin{array}{c}
\sigma_{x x}^{i} \\
\sigma_{y y}^{i} \\
\sigma_{x y}^{i} \\
\sigma_{x y}^{i}
\end{array}\right\} d z_{i},\left\{\begin{array}{c}
Q_{x z}^{i} \\
Q_{y z}^{i}
\end{array}\right\}=k_{s} \int_{-h_{i} / 2}^{h_{i} / 2}\left\{\begin{array}{c}
\sigma_{x z}^{i} \\
\sigma_{y z}^{i}
\end{array}\right\} d z_{i} ; i=t, b \text { and } n=1,2,3 .
\end{aligned}
$$

Because the face sheets are thin $\left(\frac{z_{i}}{R_{x i}}, \frac{z_{i}}{R_{y i}}<<1, i=t, b\right), \frac{z_{i}}{R_{x i}}$ and $\frac{z_{i}}{R_{y i}}$ can be neglected, therefore the stress resultants per unit length for the face sheets can be defined as follow:

$$
\left\{\begin{array}{c}
N_{x x}^{i} \\
N_{y y}^{i} \\
N_{x y}^{i} \\
N_{y x}^{i}
\end{array}\right\}=\int_{-h_{i} / 2}^{h_{i} / 2}\left\{\begin{array}{c}
\sigma_{x x}^{i} \\
\sigma_{y y}^{i} \\
\sigma_{x y}^{i} \\
\sigma_{x y}^{i}
\end{array}\right\} d z_{i},\left\{\begin{array}{c}
M_{x x}^{i} \\
M_{y y}^{i} \\
M_{x y}^{i} \\
M_{y x}^{i}
\end{array}\right\}=\int_{-h_{i} / 2}^{h_{i} / 2} z_{i}\left\{\begin{array}{c}
\sigma_{x x}^{i} \\
\sigma_{y y}^{i} \\
\sigma_{x y}^{i} \\
\sigma_{x y}^{i}
\end{array}\right\} d z_{i},\left\{\begin{array}{c}
Q_{x z}^{i} \\
Q_{y z}^{i}
\end{array}\right\}=k_{s} \int_{-h_{i} / 2}^{h_{i} / 2}\left\{\begin{array}{c}
\sigma_{x z}^{i} \\
\sigma_{y z}^{i}
\end{array}\right\} d z_{i} ; i=t, b
$$

The constitutive equations for in-plane stress resultants based on the first order shear deformation laminate theory are defined as (Reddy 2003): 


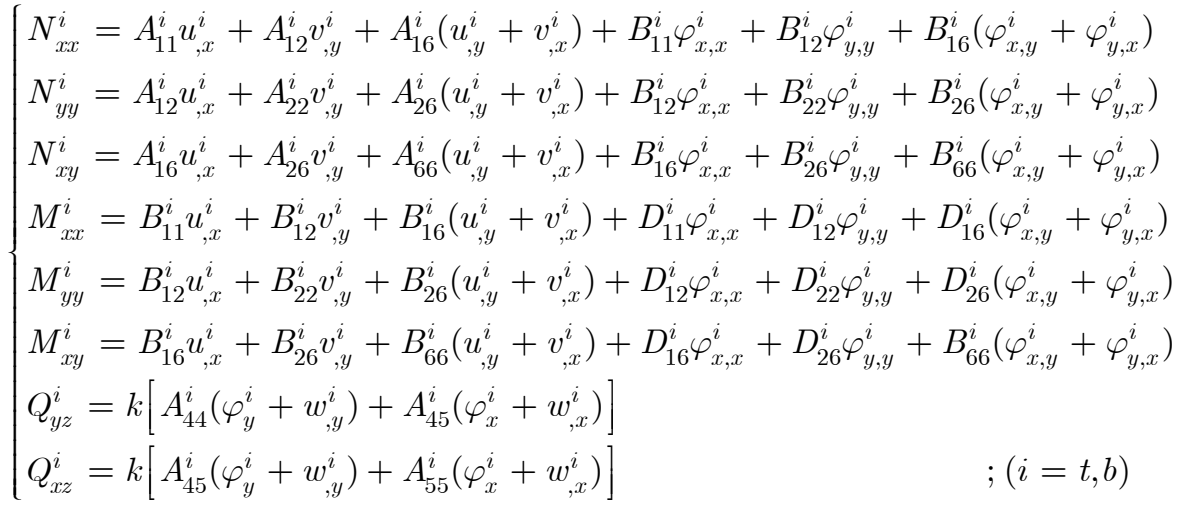

where the laminate stiffness coefficients for the face sheets are defined by:

$$
\begin{aligned}
& \left(A_{i j}, B_{i j}, D_{i j}\right)^{m}=\int_{-h_{m} / 2}^{h_{m} / 2} \bar{Q}_{i j}\left(1, z, z^{2}\right) d z_{m}=\left(\sum_{k=1}^{N} \bar{Q}_{i j}^{(k)} \int_{z_{(k)}}^{z_{(k+1)}}\left(1, z, z^{2}\right) d z\right)^{m}, \\
& A_{i j}^{m}=\left(\sum_{k=1}^{N} \bar{Q}_{i j}^{(k)}\left(z_{(k+1)}-z_{(k)}\right)\right)^{m}, \quad B_{i j}^{m}=\left(\frac{1}{2} \sum_{k=1}^{N} \bar{Q}_{i j}^{(k)}\left(z_{(k+1)}^{2}-z_{(k)}^{2}\right)\right)^{m}, \\
& D_{i j}^{m}=\left(\frac{1}{3} \sum_{k=1}^{N} \bar{Q}_{i j}^{(k)}\left(z_{(k+1)}^{3}-z_{(k)}^{3}\right)\right)^{m} \quad ; \quad i, j=1,2,6 ; m=t, b, \\
& A_{i j}^{m}=\left(\sum_{k=1}^{N} \bar{Q}_{i j}^{(k)}\left(z_{(k+1)}-z_{(k)}\right)\right)^{m} \quad ; \quad i, j=4,5 \quad ; m=t, b .
\end{aligned}
$$

where $A_{i j}, B_{i j}$ and $D_{i j}$ are called the extensional stiffness, the bending-extensional coupling stiffness and the bending stiffness, respectively.

\section{ANALYTICAL SOLUTION}

The displacement fields at the top and bottom face-sheets based on double Fourier series for a doubly curved composite sandwich panel with simply supported boundary conditions are assumed to be in the following form:

$$
\left[\begin{array}{l}
u_{0}^{j}(x, y) \\
v_{0}^{j}(x, y) \\
w_{0}^{j}(x, y) \\
\psi_{x}^{j}(x, y) \\
\psi_{y}^{j}(x, y) \\
u_{k}^{c}(x, y) \\
v_{k}^{c}(x, y) \\
w_{l}^{c}(x, y)
\end{array}\right]=\sum_{n=1}^{\infty} \sum_{m=1}^{\infty}\left[\begin{array}{c}
U_{0 m n}^{j} \cos \left(\alpha_{m} x\right) \sin \left(\beta_{n} y\right) \\
V_{0 m n}^{j} \sin \left(\alpha_{m} x\right) \cos \left(\beta_{n} y\right) \\
W_{0 m n}^{j} \sin \left(\alpha_{m} x\right) \sin \left(\beta_{n} y\right) \\
\Psi_{y m n}^{j} \sin \left(\alpha_{m} x\right) \cos \left(\beta_{n} y\right) \\
U_{k m n}^{c} \cos \left(\alpha_{m} x\right) \sin \left(\beta_{n} y\right) \\
V_{k m n}^{c} \sin \left(\alpha_{m} x\right) \cos \left(\beta_{n} y\right) \\
W_{l m n}^{c} \sin \left(\alpha_{m} x\right) \sin \left(\beta_{n} y\right)
\end{array}\right], \quad(\mathrm{k}=0,1,2,3),(\mathrm{l}=0,1,2)
$$


In Equation $(30), U_{0 m n}^{j}, V_{0 m n}^{j}, W_{0 m n}^{j}, \Psi_{x m n}^{j}, \Psi_{y m n}^{j}, U_{k m n}^{c}, V_{k m n}^{c}$ and $W_{l m n}^{c}$ are Fourier coefficients and $\mathrm{m}$ and $\mathrm{n}$ are the half wave numbers along $\mathrm{x}$ and $\mathrm{y}$ directions, respectively. The above double Fourier series functions can satisfy some boundary conditions for a doubly curved sandwich panel i.e., simply supported on all edges. However, when all edges are clamped, the functions $\cos \left(\alpha_{m} x\right)$ and $\cos \left(\beta_{n} y\right)$ in the above series expansions must be replaced with $\cos \left(\alpha_{m} x\right)$ and $\sin \left(\beta_{n} y\right)$, respectively.

In Equations (11)-(25), the static loads $\left(q_{j}(j=t, b)\right)$ normal to the top and/or bottom face sheets are assumed to be represented by the following series expansions:

$$
q_{j}(x, y)=\sum_{m=1}^{\infty} \sum_{n=1}^{\infty} q_{m n}^{j} \sin \left(\alpha_{m} x\right) \sin \left(\beta_{n} y\right) ; j=t, b
$$

where $q_{m n}$ is Fourier coefficient that dependes on the types of the loads. Fourier coefficient for the uniformly distributed load on the top and/or bottom face sheets of the double curved composite sandwich panel can be obtained as follows:

$$
\begin{gathered}
q_{m n}^{j}=\frac{4}{a b} \int_{0}^{a} \int_{0}^{b} q_{j}(x, y)\left(\sin \alpha_{m} x\right)\left(\sin \beta_{n} y\right) d x d y, q_{j}(x, y)=P_{0} ; j=t, b \Rightarrow \\
\begin{cases}q_{m n}^{j}=\frac{16 P_{0}}{m n \pi^{2}} & \text { for }(m, n=1,3,5, \ldots . .) \\
q_{m n}^{j}=0 & \text { for }(m, n=2,4,6, \ldots . .)\end{cases}
\end{gathered}
$$

and for the point load acting on an arbitrary point $\left(x_{i}, y_{i}\right)$ can be determined as follow:

$$
\begin{gathered}
q_{m n}^{j}=\frac{4}{a b} \int_{0}^{a} \int_{0}^{b} q_{j}(x, y)\left(\sin \alpha_{m} x\right)\left(\sin \beta_{n} y\right) d x d y, q_{j}(x, y)=P_{0} \delta\left(x-x_{i}\right) \delta\left(y-y_{i}\right) ; j=t, b \Rightarrow \\
q_{m n}^{j}=\frac{4 P_{0}}{a b}\left(\sin \frac{m \pi x_{i}}{a}\right)\left(\sin \frac{n \pi y_{i}}{b}\right)
\end{gathered}
$$

Fourier coefficient for the area load on the certain rectangular area $(2 u \times 2 v)$ of the top and/or bottom face sheets of the double curved composite sandwich panels can be written as follow:

$$
\begin{gathered}
q_{m n}^{j}=\frac{4}{a b u v} \int_{x_{i}-u}^{x_{i}+u \int_{i}-v} q_{j}(x, y)\left(\sin \alpha_{m} x\right)\left(\sin \beta_{n} y\right) d x d y, q_{j}(x, y)=q_{0} \delta\left(x-x_{i}\right) \delta\left(y-y_{i}\right) ; j=t, b \Rightarrow \\
q_{m n}^{j}=\frac{16 q_{0}}{m n u v \pi^{2}}\left(\sin \frac{m \pi x_{i}}{a}\right)\left(\sin \frac{n \pi y_{i}}{b}\right)\left(\sin \frac{m \pi u}{2 a}\right)\left(\sin \frac{n \pi v}{2 b}\right)
\end{gathered}
$$

and for the harmonic load on the top and/or bottom sheets, it can be determined as follows: 


$$
\begin{gathered}
q_{m n}^{j}=\frac{4}{a b} \int_{0}^{a} \int_{0}^{b} q_{j}(x, y)\left(\sin \alpha_{m} x\right)\left(\sin \beta_{n} y\right) d x d y, q_{j}(x, y)=q_{0}\left(\sin \frac{\pi x}{a}\right)\left(\sin \beta \frac{\pi y}{b}\right) ; j=t, b \Rightarrow \\
q_{m n}^{j}=q_{0} \text { only }(m=n=1)
\end{gathered}
$$

Therefore, the governing equation of motion to the bending analysis can be written as follows:

$$
\begin{gathered}
{[K]\{c\}=\{Q\}} \\
\{c\}=\left\{U_{0 m n}^{t}, U_{0 m n}^{b}, V_{0 m n}^{t}, V_{0 m n}^{b}, W_{0 m n}^{t}, W_{0 m n}^{b}, \psi_{x m n}^{t}, \psi_{x m n}^{b}, \psi_{y m n}^{t}, \psi_{y m n}^{b}, U_{0 m n}^{c}, V_{0 m n}^{c}, U_{1 m n}^{c}, V_{1 m n}^{c}, W_{0 m n}^{c}\right\}^{T} \\
\{Q\}=\left\{0,0,0,0,-q_{m n}^{t}, q_{m n}^{b}, 0,0,0,0,0,0,0,0,0\right\}^{T}
\end{gathered}
$$

where $[\mathrm{K}]$ is the $(15 \mathrm{mn}) \times(15 \mathrm{mn})$ stiffness matrix and, for SSSS and CCCC B.Cs., some of matrix elements are given in Appendix A and $[\mathrm{Q}]$ is the $(15 \mathrm{mn}) \times(1)$ vector of the arbitrary static force $(\mathrm{s})$.

\section{RESULTS AND DISCUSSION}

In this section, some examples are considered and the obtained results are validated and discussed. To validate the present results and demonstrate their capability in predicting the static bending analysis of a doubly curved composite sandwich panel, some examples are presented and the results obtained from the present theory are compared with the recent theoretical and numerical results found in the literature. Since, there is no research about the bending analysis of a doubly curved sandwich structure to validate the obtained results, sandwich structures were modeled in ABAQUS FE code and the results from the analytical formulations were compared with FE code. The agreement between the results was quite good.

\subsection{Static bending analysis of flat composite sandwich panel with SSSS B.C.}

In this example, the bending analysis of a flat composite sandwich panel with SSSS B.C. was investigated. Mechanical properties of the core and the face sheets are given in Table 1 . The face sheet to panel thickness ratio was 0.1 and the core to panel thickness ratio was 0.8 (Pandit et al., 2008). The top face sheet of the sandwich panel was subjected to the harmonic load $\left(q_{t}(x, y)=q_{0}(\sin p x / a)(\sin p y / b)\right)$. Results of the dimensionless deflection of central mid-plane of the core $\left(\bar{w}=100 w E h^{3} / q a^{4}, z_{c}=0\right)$ for different panel thickness to length ratios (h/a) were compared with those of the presented formulations (IHSAPT) and the results obtained from 3D elasticity solution (Pandit et al., 2008) in Table 2. As can be seen in Table 2, there was quite good agreement between the results and there was a little difference between them. Note that, the 3D elasticity solution was an exact solution.

Table 1 Material properties of a flat composite sandwich panel (Pandit et al., 2008)

\begin{tabular}{cc}
\hline \hline $\begin{array}{c}\text { Face sheets } \\
\text { core }\end{array}$ & $\mathrm{E}_{1}=25 \mathrm{E}, \mathrm{E}_{2}=\mathrm{E}, \mathrm{G}_{12}=\mathrm{G}_{13}=0.5 \mathrm{E}, G_{23}=0.2 \mathrm{E}, \nu_{12}=0.25$. \\
\hline \hline
\end{tabular}


Table 2 Comparison of the dimensionless central deflection of a sandwich panel for different $\mathrm{h} / \mathrm{a}$ ratios.

\begin{tabular}{cccc}
\hline \hline & \multicolumn{3}{c}{ Dimensionless central deflection of a sandwich panel } \\
$\mathrm{h} / \mathrm{a}$ & Present model & 3D elasticity (Pandit et al. 2008) & Error difference (\%) \\
0.01 & 0.8932 & 0.8923 & -0.1 \\
0.02 & 0.9357 & 0.9348 & -0.096 \\
0.05 & 1.2278 & 1.2264 & -0.11 \\
\hline \hline
\end{tabular}

\subsection{Bending analysis of open single curved sandwich panel with SSSS B.C.}

In this example, the static bending of an open single curved sandwich panel subjected to harmonic load on the top face sheet with SSSS B.C. was studied. The face sheet to panel thickness ratio and the core to panel thickness ratio were 0.1 and 0.8 , respectively, and the properties of the composite face sheets and the foam core are given in Table 3.

Table 3 Material properties of a flat composite sandwich panel (Khare et al., 2005)

\begin{tabular}{ccc}
\hline \hline Face sheets $\quad \mathrm{E}_{1}=172.368 \mathrm{GPa}, \mathrm{E}_{2}=\mathrm{E}_{3}=6.895 \mathrm{GPa}, \mathrm{G}_{12}=\mathrm{G}_{13}=3.447 \mathrm{GPa}, \mathrm{G}_{23}=1.379 \mathrm{GPa}$, \\
\\
$\begin{array}{c}\nu_{12}=\nu_{13}=\nu_{23}=0.25 . \\
\mathrm{E}\end{array}$ \\
$\mathrm{E}_{1}=\mathrm{E}_{2}=0.276 \mathrm{GPa}, \mathrm{E}_{3}=3.447 \mathrm{GPa}, \mathrm{G}_{12}=0.110 \mathrm{GPa}, \mathrm{G}_{13}=\mathrm{G}_{23}=0.414 \mathrm{GPa}$, \\
$\nu_{12}=\nu_{13}=0.25, \nu_{23}=0.02$.
\end{tabular}

In Table 4, the dimensionless central deflection of an open single curved sandwich panel for different $R_{c} / h$ and $h / a$ ratios is presented and the results of the presented formulations (IHSPT) are compared with those of higher order equivalent single layer theory (HSDT-ESL) (Khare et al. 2005). Table 4 showed that, for a thin single curved sandwich panel, there was good agreement between the results of IHSPT and HSDT-ESL; but, they were a little different for the panels with more thickness $(\mathrm{h} / \mathrm{a}=0.25)$. Also, it can be seen in this table that the dimensionless deflection obtained from IHSPT was more than the one from HSDT-ESL and the error difference between the results for $\mathrm{h} / \mathrm{a}=0.25$ was larger than those for $\mathrm{h} / \mathrm{a}=0.1$. The reason for this difference can be that the current method can rather accurately model the flexibility of the core; in addition, Khare et al. (2005) used equivalent single layer theory, in which by increasing $\mathrm{h} / \mathrm{a}$ ratio, the error of this method increased. 
Table 4 Comparison of the dimensionless central deflection of a sandwich panel for different $R_{c} / h$ and $h / a$ ratios.

\begin{tabular}{ccccc}
\hline \hline & & \multicolumn{3}{c}{$\bar{w}=100 w E_{2} h^{3} / q a^{4}$} \\
$\mathrm{~h} / \mathrm{a}$ & $\mathrm{R}_{\mathrm{c}} / \mathrm{h}$ & Present model & HSDT-ESL(Khare et al., 2005) & Error difference (\%) \\
& 100 & 2.19925 & 2.14545 & -2.5 \\
0.1 & 50 & 2.18730 & 2.10714 & -3.8 \\
& 20 & 2.10855 & 2.09559 & -0.61 \\
& 100 & 7.62635 & 7.34982 & -3.76 \\
0.25 & 50 & 7.62569 & 7.22810 & -5.5 \\
& 20 & 7.62486 & 7.19029 & -6.04 \\
\hline \hline
\end{tabular}

\subsection{The bending of a flat composite sandwich panel with CCCC B.C.}

In this example, the static bending of a flat sandwich panel with CCCC B.C. was studied. The top face sheet of the sandwich panel was subjected to the uniformly distributed load (UDL) $\left(q_{t}(x, y)=q_{0}=1000 \mathrm{pa}\right)$ and the harmonic distributed load $(\mathrm{SSL})\left(q_{t}(x, y)=q_{0}(\sin \pi x / a)(\sin \pi y / b)\right)$. Also, the static load (q0) can be uniformly applied over an area (ULA) ( $A=2 U \times 2 V, U=V=a / 3, a=b)$. The lay-up sequences of the top and bottom face sheets were $[0 / 90 / 0]$ and the sandwich panel was symmetric about the mid-plane. Mechanical properties of the composite face sheets and the PVC foam core are given in Table 5.

The convergence of the central deflection of the top face sheet in the composite sandwich panel is presented in Table 6 . This table shows that the obtained results for sandwich panel with $\mathrm{h} / \mathrm{a}=0.1$ and $\mathrm{h}_{\mathrm{c}} / \mathrm{h}=0.88$ converged after 361 expressions $(\mathrm{m}=\mathrm{n}=19)$.

In Table 7, the results of the presented formulations were validated with those of ABAQUS analysis and reasonably good agreement was found between them. In Figure 2, the 3D view of deflection of the composite sandwich panel subjected to the uniform and harmonic distributed loads on the top face sheet obtained from ABAQUS is presented.

Table 5 Material properties of a composite sandwich panel (Meunier and Shenoi, 1999)

\begin{tabular}{cc}
\hline \hline Face sheets & $\mathrm{E}_{1}=\mathrm{E}_{2}=\mathrm{E}_{3}=0.10363 \mathrm{GPa}, \mathrm{G}_{12}=\mathrm{G}_{13}=\mathrm{G}_{23}=0.05 \mathrm{GPa}, \nu=0.32$. \\
core & $\mathrm{E}_{1}=24.51 \mathrm{GPa}, \mathrm{E}_{2}=\mathrm{E}_{3}=7.77 \mathrm{GPa}, \mathrm{G}_{12}=\mathrm{G}_{13}=3.34 \mathrm{GPa}, \mathrm{G}_{23}=1.34 \mathrm{GPa}$, \\
$\nu_{12}=\nu_{13}=0.078, \nu_{23}=0.49$. \\
\hline \hline
\end{tabular}


Table 6 The convergence of the dimensionless central deflection of the top face sheet of the composite sandwich panel subjected to the UDL and the SSL on the top face sheet

\begin{tabular}{ccc}
\hline \hline \multicolumn{3}{c}{$\bar{w}=100 w E_{2} h^{3} / q a^{4}$} \\
Convergence $(\mathrm{m}=\mathrm{n})$ & $\bar{w}(\mathrm{SSL})$ & $\bar{w}(\mathrm{UDL})$ \\
5 & 8.6156 & 11.5431 \\
9 & 9.3150 & 12.8354 \\
11 & 9.5189 & 13.2965 \\
15 & 9.6302 & 13.5085 \\
17 & 9.6627 & 13.5522 \\
19 & 9.6986 & 13.6446 \\
21 & - & 13.6852 \\
\hline \hline
\end{tabular}

Table 7 Dimensionless central deflection of the top face sheet of the composite sandwich panel subjected to the UDL and the SSL on the top face sheet

\begin{tabular}{cccc}
\hline \hline & \multicolumn{3}{c}{$\bar{w}=100 w E_{2} h^{3} / q a^{4}$} \\
Type of loads & Present model & ABAQUS & Error difference (\%) \\
UDL & 13.6852 & 13.9793 & 2.14 \\
SSL & 9.6986 & 9.8506 & 1.1 \\
ULA & 17.2609 & 17.7312 & 2.34 \\
\hline
\end{tabular}

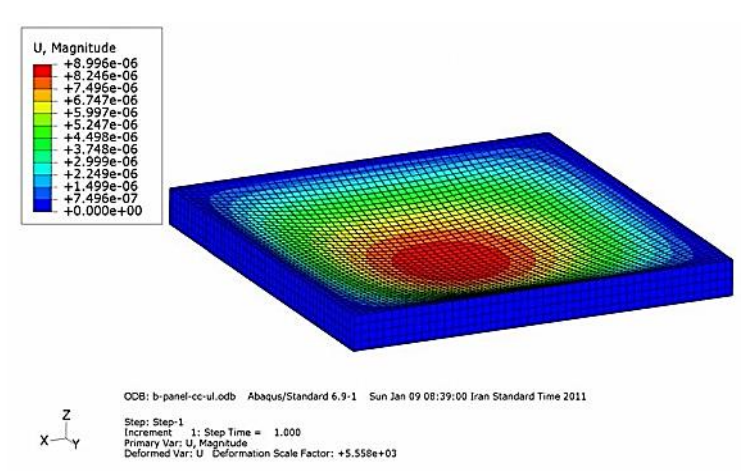

a. UDL on the top face sheet

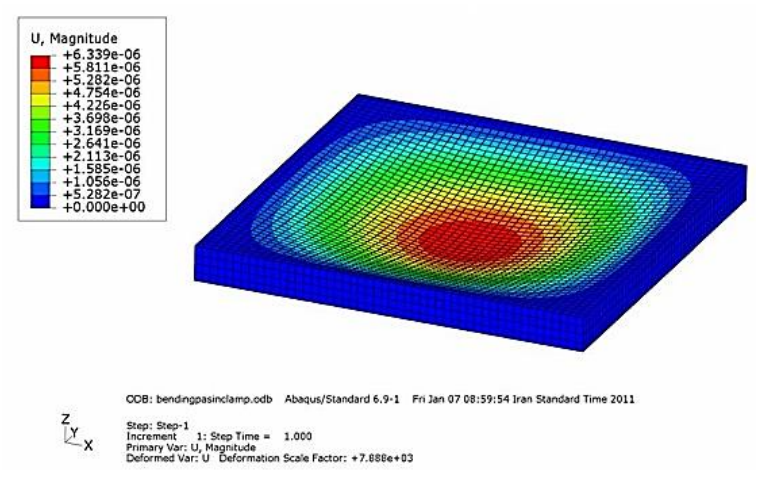

b. SSL on the top face sheet

Figure 2 3D view of deflection of the composite sandwich panel subjected to the UDL and the SSL on the top face sheet with CCCC B.C.

\subsection{The bending analysis of a double curved composite sandwich panel}

In this example, the static bending analysis of a double curved composite sandwich panel with SSSS and CCCC B.Cs. was investigated. The properties of the sandwich structure are given in Table 8. The lay-up sequences of the top and bottom face sheets were [0/90/0] and the sandwich panel was symmetric about the mid-plane. The top face sheet of the sandwich panel was subjected to the uniform distributed load (UDL) and harmonic distributed load (SSL). Also, the static load ( $\mathrm{q}_{0}$ ) was uniformly applied over an area (ULA) $(A=2 U ¥ 2 V, U=V=a / 8)$. 
In Table 9, the dimensionless deflections at the center of the face sheets and the core converging after 361 expressions $(\mathrm{m}=\mathrm{n}=19)$ are presented for both boundary conditions. It is obvious from the table that the dimensionless deflections for SSSS B.C. were more than those for CCCC B.C., because the flexibility of the sandwich structure with SSSS B.C. was more than that with CCCC B.C. The dimensionless deflection at the center of the bottom face sheet was less than that at the center of top face sheet, because the flexibility of the core was modeled, which caused the deflections of the top and bottom face sheets to be different. In this analysis, the static load was $q_{0}=10 M P a$ and thicknesses of the face sheets was $\mathrm{h}=3 \mathrm{~mm}$.

Table 8 Mechanical and geometrical properties of a double curved composite sandwich panel.

\begin{tabular}{cc}
\hline \hline Face sheets & $\mathrm{E}_{1}=\mathrm{E}_{2}=\mathrm{E}_{3}=0.10363 \mathrm{GPa}, \mathrm{G}_{12}=\mathrm{G}_{13}=\mathrm{G}_{23}=0.05 \mathrm{GPa}, n=0.32$. \\
Core & $\mathrm{E}_{1}=24.51 \mathrm{GPa}, \mathrm{E}_{2}=\mathrm{E}_{3}=7.77 \mathrm{GPa}, \mathrm{G}_{12}=\mathrm{G}_{13}=3.34 \mathrm{GPa}, \mathrm{G}_{23}=1.34 \mathrm{GPa}$, \\
& $n_{12}=n_{13}=0.078, n_{23}=0.49$. \\
Geometric & $h_{c} / h=0.88, a=10 h, R_{c 1}=R_{c 2}=3 a, a=b$. \\
\hline \hline
\end{tabular}

Table 9 Dimensionless deflections at center of the face sheets and the core of a double curved composite sandwich panel subjected to the uniform distributed load (UDL), the harmonic distributed load (SSL) and the uniform static load applied over an area (ULA).

\begin{tabular}{ccccccc}
\hline \hline & \multicolumn{5}{c}{$\bar{w}(a / 2, b / 2,0)=100 w E_{2 t} h^{3} / q R_{t}^{4}$} \\
Load types & \multicolumn{5}{c}{ SSSS B.C. } & \multicolumn{3}{c}{ CCCC B.C. } \\
& $\bar{w}_{t}$ & $\bar{w}_{c}$ & $\bar{w}_{b}$ & $\bar{w}_{t}$ & $\bar{w}_{c}$ & $\bar{w}_{b}$ \\
UDL & 1.6056 & 0.9241 & 0.2155 & 1.6068 & 0.9239 & 0.2146 \\
SSL & 4.8231 & 3.5157 & 2.1820 & 4.7406 & 3.4404 & 2.1135 \\
ULA & 9.4353 & 7.6133 & 6.2797 & 8.7950 & 6.9700 & 5.6192 \\
\hline \hline
\end{tabular}

\subsection{Effect of some important parameters on the static response of a double curved sand- wich panel}

In this example, the effects of types of boundary conditions, types of applied loads, core to panel and radii curvatures ratios on the bending response of a double curved composite sandwich panel were investigated. The properties of the sandwich structure are given in Table 8 . The lay-up sequences of the top and bottom face sheets were [0/90/0] and the sandwich panel was symmetric about the mid-plane. The top face sheet of the sandwich panel was subjected to the uniform distributed load (UDL), harmonic distributed load (SSL), and point load (PL). Also, the static load $\left(q_{0}=-10 M P a\right)$ was uniformly applied over an area (ULA) $(A=2 U \times 2 V, U=V=a / 8)$.

Variations of deflections of the face sheets with the variation of the core to the panel thickness ratio for a double curved sandwich panel subjected to PL and UDL for SSSS and CCCC B.Cs. are presented in Figure 3. As demonstrated in Figure 3, with increasing the $\mathrm{h}_{\mathrm{c}} / \mathrm{h}$ ratio, the dimensionless deflections of the top and bottom face sheets increased in all cases. For the low core to the panel thickness ratio $\left(h_{c} / h\right)$, the increasing rate of deflection was low, while by increasing the ratio, that rate increased. Also in all the cases, deflection of the top and bottom face sheets subjected to PL was higher than those subjected to UDL. The dimensionless deflection at the center 
of the bottom face sheet was lower than that at the center of the top face sheet, for the same reason mentioned above. Variations in deflections of the face sheets with radii curvatures ratio $\left(R_{2} / R_{1}\right)$ for a double curved sandwich panel subjected to SSL and UDL for SSSS and CCCC B.Cs. are presented in Figure 4.

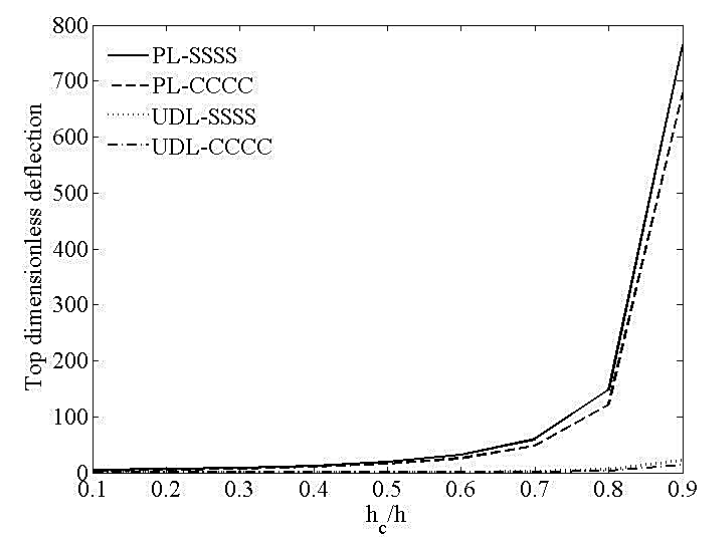

(a) Top face sheet deflection

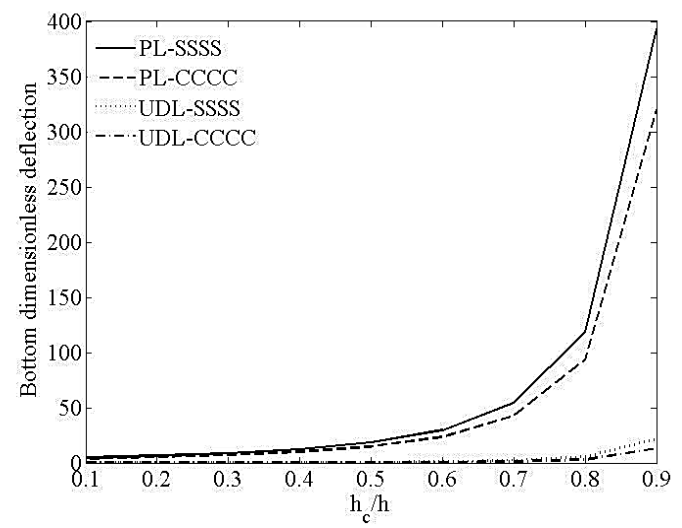

(b) Bottom face sheet deflection

Figure 3 The variations of the face sheets deflections with core to panel thickness ratio for a double curved sandwich panel subjected to PL and UDL for SSSS and CCCC B.Cs.

As can be seen in Figure 4, with increasing ratio of radii curvatures, deflections of the top and bottom face sheets increased in all the cases. For low radii curvatures ratio $\left(R_{2} / R_{1}\right)$, increase in the rate of the deflection was high, while by increasing the $R_{2} / R_{1}$ ratio, the rate decreased. Also, this figure showed that, in each $\mathrm{R}_{2} / \mathrm{R}_{1}$ ratio, the top and bottom face sheet deflections for SSSS B.C. were higher than those for CCCC B.C. and maximum deflections occurred in the sandwich panel subjected to UDL with SSSS B.C. By increasing the $R_{2} / R_{1}$ ratio, the flexibility of the sandwich panel slightly increased, which caused the deflections of the top and bottom face sheets to increase. In Figure 5, variation in deflections of the top and bottom face sheets along the x-axis for a double curved composite sandwich panel subjected to SSL and UAL for SSSS and CCCC B.Cs. is presented.

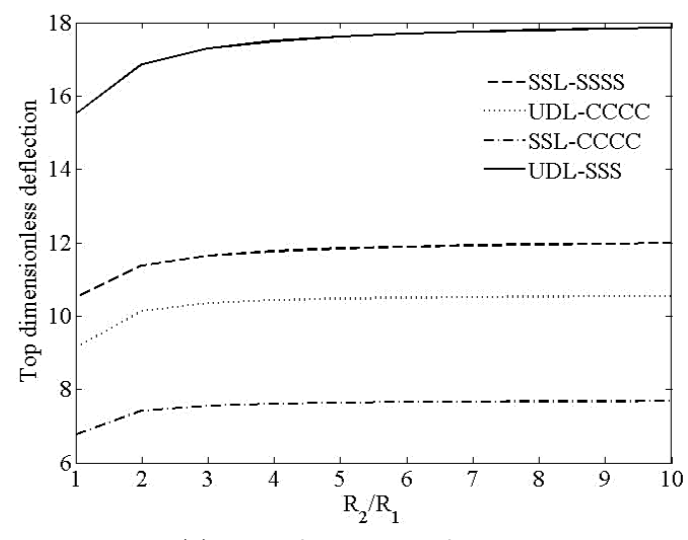

(a) Top face sheet deflection

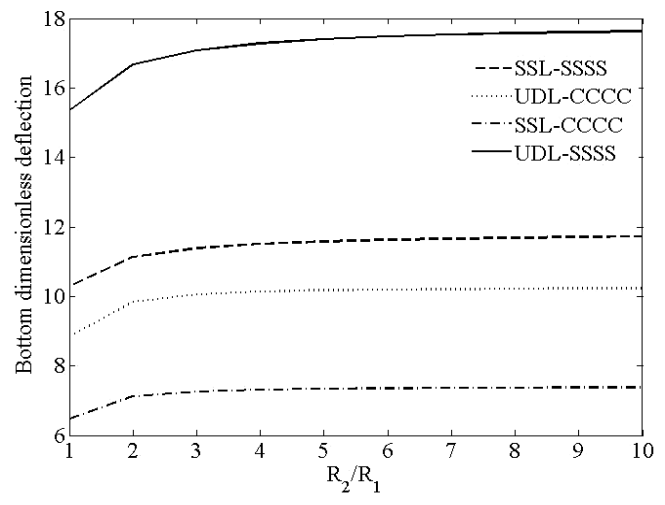

(b) Bottom face sheet deflection

Figure 4 The variations of the face sheets deflections with radii curvatures ratio for a double curved sandwich panel subjected to SSL and UDL for SSSS and CCCC B.Cs. 


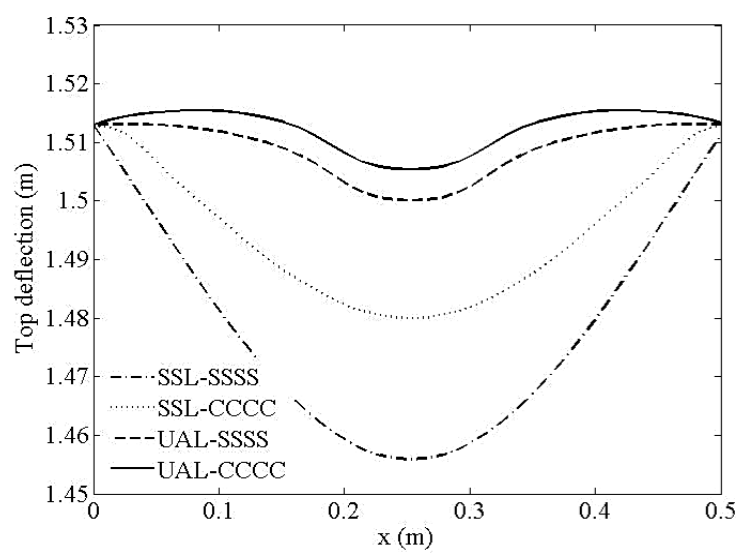

(a) The top face sheet deflection

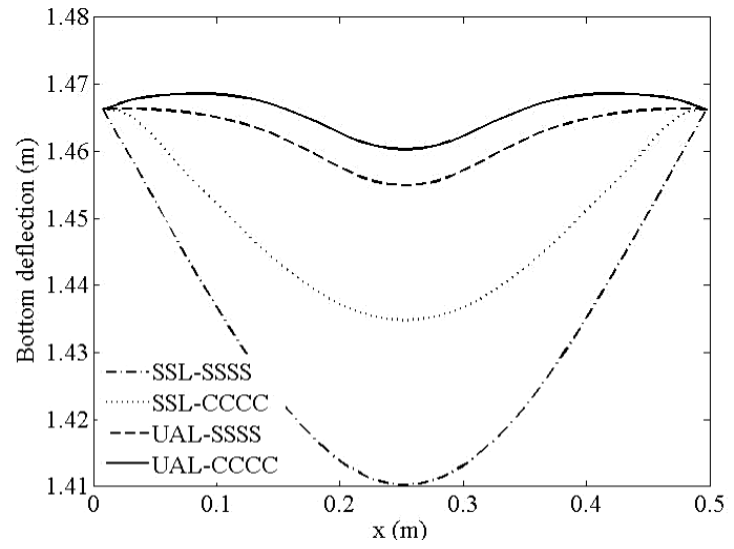

(b) The bottom face sheet deflection

Figure 5 The top and bottom face sheet deflections for a double curved composite sandwich panel subjected to SSL and UAL for SSSS and CCCC B.Cs.

As is obvious from Figure 5, in all the cases, the top face sheet deflection was higher than the bottom face sheet deflection, which was due to the flexibility of the core. The maximum deflections of face sheets occurred in the sandwich panel subjected to UDL with SSSS B.C.. Also, Figure 5 shows that, when the sandwich panel with both boundary conditions was subjected to UAL (i.e. the static load on a patch), only the area under the applied load was approximately deflected, while for the sandwich panel subjected to SSL, deflection occurred on the entire panel.

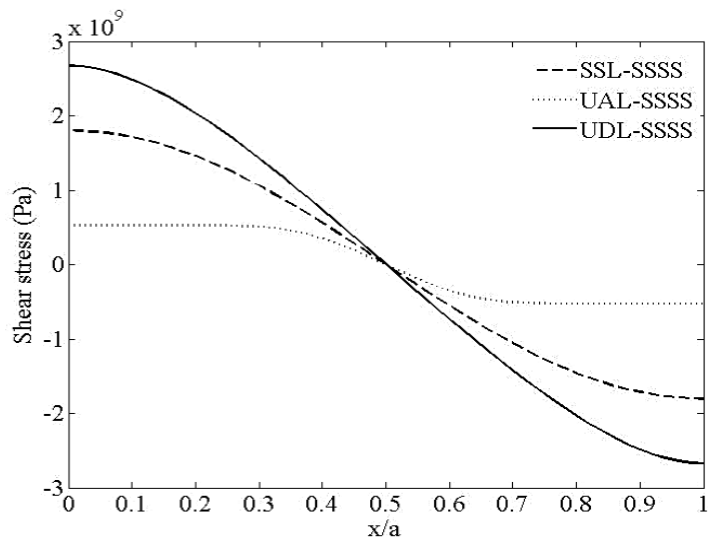

(a) SSSS B.C

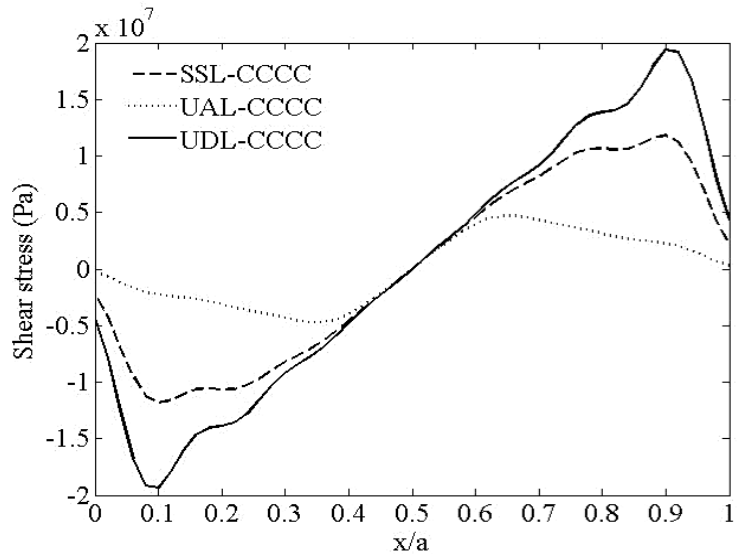

(b) CCCC B.C.

Figure 6 The variation of the shear stress along $x$-axis at the top face sheet-core interface of a double curved composite sandwich panel for SSSS and CCCC B.Cs. subjected to UDL, UAL and SSL

In Figure 6, variation in the shear stress at the top face sheet-core interface of a double curved composite sandwich panel with both types of boundary conditions subjected to UDL, UAL, and SSL is presented.

Figure 6(a)-(b) shows that maximum shear stress of a double curved composite sandwich panel with SSSS and CCCC B.Cs. subjected to UDL was higher than that subjected to other types of 
applied loads. Also, Figure 6(a) shows that maximum shear stress for all types of applied loads occurred at the edges of the panel $(\mathrm{x} / \mathrm{a}=0,1 ; \mathrm{y}=\mathrm{b} / 2)$. But, it can be seen in Figure $6(\mathrm{~b})$ that maximum shear stress did not occur at the edges of the panel and occurred at $\mathrm{x} / \mathrm{a}=0.1$ or $\mathrm{x} / \mathrm{a}=0.9$ when the sandwich panel was subjected to SSL and UDL. The maximum shear stress occurred at $\mathrm{x} / \mathrm{a}=0.37$ or $\mathrm{x} / \mathrm{a}=0.63$ in the case of the sandwich panel subjected to UAL. The shear stress at the edges and maximum shear stresses for all types of the applied loads and for both boundary conditions are given in Table 10.

Table 10 The shear stress at the edges and the maximum shear stresses for all types of applied loads and for SSSS and CCCC B.Cs.

\begin{tabular}{|c|c|c|c|c|}
\hline \multirow{2}{*}{$\begin{array}{c}\text { Types of } \\
\text { B.Cs. }\end{array}$} & \multirow{2}{*}{$\begin{array}{c}\text { Types of } \\
\text { applied loads }\end{array}$} & \multicolumn{2}{|c|}{ The shear stress at the edges $(\mathrm{Pa})$} & \multirow{2}{*}{$\begin{array}{c}\text { The maximum } \\
\text { shear } \\
\text { stress }(\mathrm{Pa})\end{array}$} \\
\hline & & $\mathrm{x}=0, \mathrm{y}=\mathrm{b} / 2$ & $\mathrm{x} / \mathrm{a}=1, \mathrm{y}=\mathrm{b} / 2$ & \\
\hline \multirow{3}{*}{ SSSS } & UDL & $2.65 \times 10^{9}$ & $-2.65 \times 10^{9}$ & $2.65 \times 10^{9}$ \\
\hline & SSL & $1.8 \times 10^{9}$ & $-1.8 \times 10^{9}$ & $1.8 \times 10^{9}$ \\
\hline & $\mathrm{UAL}$ & $0.5 \times 10^{9}$ & $-0.5 \times 10^{9}$ & $0.5 \times 10^{9}$ \\
\hline \multirow{3}{*}{$\mathrm{CCCC}$} & UDL & $-0.49 \times 10^{7}$ & $0.49 \times 10^{7}$ & $-1.9 \times 10^{7}$ \\
\hline & SSL & $-0.25 \times 10^{7}$ & $0.25 \times 10^{7}$ & $-1.15 \times 10^{7}$ \\
\hline & UAL & $-0.05 \times 10^{7}$ & $0.05 \times 10^{7}$ & $-0.48 \times 10^{7}$ \\
\hline
\end{tabular}

Finally, the effects of various types of applied loads and boundary conditions on the normal stress were investigated. The variation of normal stress $\left(\sigma_{z z}^{c}\right)$ along the core thickness (z-axis) for various types of applied static loads and boundary conditions is shown in Figure 7. This figure demonstrates that the normal stress increased in all the cases along the core thickness from the top face sheet-core interface to the bottom face sheet-core interface $\left(z_{c} / h_{c}=0.5\right)$. Also, it can be seen that maximum normal stress at the bottom face sheet-core interface occurred in the case of sandwich panel subjected to UDL with CCCC B.C. (-0.95 MPa) and at the top face sheet-core interface occurred in the case of sandwich panel subjected to UAL with CCCC B.C.. The minimum and maximum normal stresses across the core thickness occurred in the cases of sandwich panel subjected to UDL with SSSS B.C. and subjected to UAL with CCCC B.C., respectively. 


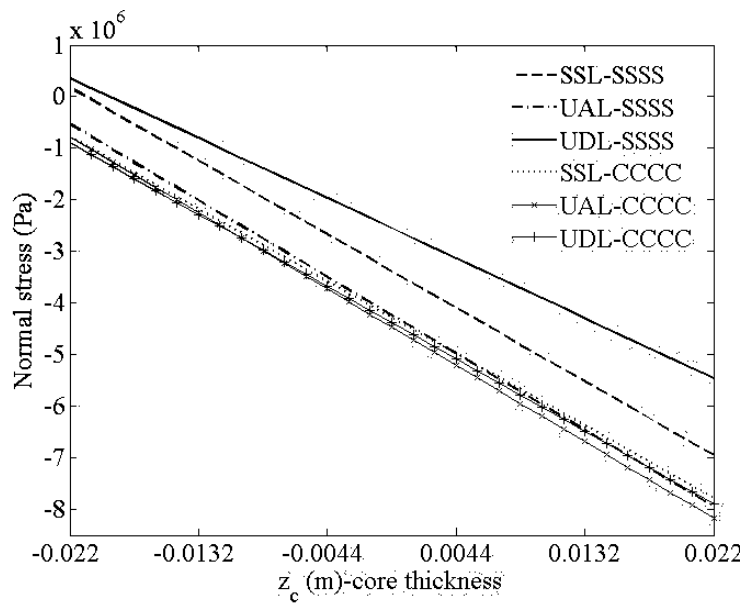

Figure 7 The variation of normal stress along the core thickness for various types of applied static loads and boundary conditions

\section{CONCLUSION}

For the first time in this study, the static bending analysis of a double curved composite sandwich panel subjected to various types of applied static loads with SSSS and CCCC B.Cs. was studied. Using the improved higher order sandwich plate theory, the governing equations on the doubly curved sandwich panel awee derived based on the principle of minimum potential energy. Since the cross-sectional warping was accurately modeled by this theory, it did not require any shear correction factor. Also, the present analysis incorporated trapezoidal shape factor (the $1+\mathrm{z} / \mathrm{R}$ terms) of a curved panel element that arose due to the fact that stresses over the panel thickness were to be integrated on cross-section of a curved panel element to obtain accurate stressresultants. The solutions were also applicable to flat panels by taking radius of curvature as infinity. The solution procedure was validated by comparing its results with those obtained either numerically or analytically by other researchers in the literature. Geometries were used in the present work for the consideration of different radii of curvatures of the face sheets and the core was unique. The general formulation which can be used for a wide range of geometries such as open single curved, flat composite, and cylindrical circular sandwich panels was presented. In conclusion, the effect of boundary conditions, core to panel thickness ratio, and radii curvature ratio on the bending analysis of the double curved composite sandwich panel was studied in detail.

\section{References}

Kheirikhah, M.M., Khalili, S.M.R. and Malekzadeh Fard, K. (2011). Biaxial buckling analysis of soft-core composite sandwich plates using improved high-order theory, European Journal of Mechanics A/Solids 31: 54-66.

Frostig, Y. and Shenar, Y. (1995). High-order bending of sandwich beams with a transversely flexible core and unsymmetrical laminated composite skins, Composite Engineering 5(4): 405-414.

Frostig, Y. and Baruch, M. (1996). Localized load effects in high-order bending of sandwich panels with flexible core, Journal of Engineering Mechanics 122 (11): 1069-1076. 
Frostig, Y. and Thomsen, O.T. (2004). Higher-order free vibration of sandwich panels with a flexible core, International Journal of Solids and Structures 41: 1697-1724.

Bozhevolnaya, E. and Frostig, Y. (1997). Nonlinear closed-form high-order analysis of curved sandwich panels, Composire Struclures 38: 383-394.

Hause, T., Librescu L. and Camarda, C.J. (1998). Postbuckling of anisotropic flat and doubly-curved sandwich panels under complex loading conditions, International Journal of Solids and Structures 35(23): 3007-3027.

Frostig, Y. (1999). Bending of curved sandwich panels with transversely flexible cores- closed-form high-order theory, Journal of Sandwich Structures and Materials 1: 4-41.

Petras, A. and Sutcliffe, M.P.F. (1999). Indentation resistance of sandwich beams, Composite Structures, 46(4), $413-424$.

Malekzadeh, K., Khalili, M.R. and Mittal, R.K. (2005a). Local and global damped vibrations of plates with a viscoelastic soft flexible core: an improved high-order approach, Journal of Sandwich Structures and Materials7: 431-456.

Zenkour, A.M. (2005a). A comprehensive analysis of functionally graded sandwich plates: part 1 - deflection and stresses, International Journal of Solids and Structures 42: 5224-42.

Zenkour, A.M. (2005b). A comprehensive analysis of functionally graded sandwich plates: part 2 - deflection and stresses, International Journal of Solids and Structures 42: 5224-42.

Hohe, J., Librescu, L. and Oh, S.Y. (2006). Dynamic buckling of flat and curved sandwich panels with transversely compressible core, Composite Structures 74: 10-24.

Garg, A.K., Kharem, R.K. and Kant, T. (2006). Higher-order closed-form solutions for free vibration of laminated composite and sandwich shells, Journal of Sandwich Structures and Materials 8: 205-35.

Kapuria, S., Bhattacharyya, M. and Kumar, A.N. (2008). Bending and free vibration response of layered functionally graded beams: a theoretical model and its experimental validation, Composite Structures 82: 390-402.

Rahmani, O., Khalili, S.M.R. and Malekzadeh, K. (2009). Free vibration response of composite sandwich cylindrical shell with flexible core, Composite Structures 92: 1269-1281.

Carrera, E. and Brischetto, S. (2009). A comparison of various kinematic models for sandwich shell panels with soft core, Journal of Composite Materials 43: 2201-2221.

Cetkovic, M.D. and Vuksanovic, j. (2009). Bending, free vibrations and buckling of laminated composite and sandwich plates using a layerwise displacement model, Composite Structures 88: 219-227.

Shen, J., Lu, G., Wang, Z. and Zhao, L. (2010). Experiments on curved sandwich panels under blast loading, International Journal of Impact Engineering 37: 960-970.

Biglari, H. and Jafari, A.A. (2010). Static and free vibration analyses of doubly curved composite sandwich panels with soft core based on a new three-layered mixed theory, Journal of Mechanical Engineering Science 224: 2332-2349.

Zhen, W. and Wanji, C. (2010). A C $\mathrm{C}^{0}$-type higher-order theory for bending analysis of laminated composite and sandwich plates, Composite Structures 92: 653-661.

Stürzenbecher, R. and Hofstetter, K. (2011). Bending of cross-ply laminated composites: An accurate and efficient plate theory based upon models of Lekhnitskii and Ren, Composite Structures 93: 1078-1088.

He, L., Cheng, Y.S. and Liu J. 2012. Precise bending stress analysis of corrugated-core, honeycomb-core and Xcore sandwich panels, 94 (5) 1656-1668.

Khedmati, M.R., Sangtabi, M.R., and Fakoori, M. (2013). Stacking sequence optimisation of composite panels subjected to slamming impact loads using a genetic algorithm, Latin American Journal of Solids \& Structures 10: $1043-1060$. 
Neto, A.T., de Silva Bussamra, F.L. and de Castro e Silva, H.A. (2014). A new metamodel for reinforced panels under compressive loads and its application to the fuselage conception, Latin American Journal of Solids \& Structures 11: 223-244.

Reddy, J.N. (2003). Mechanics of Laminated Composite Plates and Shells: Theory and Analysis, Second Edition. CRC Press.

Pandit, M.K., Sheikh, A.H. and Singh, B.N. (2008). An improved higher order zigzag theory for the static analysis of laminated sandwich plate with soft core, Finite Element in Analysis and Design44: 602 - 610 .

Khare, R.K., Rode, V., Garg, A.K. and John, S.P.H. (2005). Higher-order closed-form solutions for thick laminated sandwich shells, Journal of Sandwich Structures and Materials 7: 335-358.

Meunier, M. and Shenoi, R.A. (1999). Free vibration analysis of composite sandwich plates ,Journal of Mechanical Engineering Science 213(7): 715-727.

\section{Appendix A}

Some stiffness matrix coefficients for SSSS B.C.

$$
\begin{aligned}
& K(3,2)=\frac{a b}{4}\left[\alpha_{p} \beta_{q}\left[-4 \frac{g_{4}^{c y x}}{h_{c}^{4}}-8 \frac{g_{5}^{c y x}}{h_{c}^{5}}-16 \frac{g_{6}^{c y x}}{h_{c}^{6}}\right],\right. \\
& K(8,10)=\frac{a b}{4}\left[\alpha_{p} \beta_{q} h_{b}^{2}\left[-\frac{g_{4}^{c y x}}{h_{c}^{4}}+4 \frac{g_{5}^{c y x}}{h_{c}^{5}}-4 \frac{g_{6}^{c y x}}{h_{c}^{6}}\right]\right], \\
& K(13,7)=\frac{a b}{4}\left[\alpha_{p} \beta_{q} h_{t}\left[\frac{g_{2}^{c x z}}{h_{c}^{2}}+2 \frac{g_{3}^{c x z}}{h_{c}^{3}}-4 \frac{g_{4}^{c x z}}{h_{c}^{4}}-8 \frac{g_{5}^{c x z}}{h_{c}^{5}}+\frac{g_{3}^{c x z}}{R_{c y} h_{c}^{2}}+2 \frac{g_{4}^{c x z}}{R_{c y} h_{c}^{3}}-4 \frac{g_{5}^{c x z}}{R_{c y} h_{c}^{4}}-8 \frac{g_{6}^{c x z}}{R_{c y} h_{c}^{5}}\right]\right] .
\end{aligned}
$$

Some stiffness matrix coefficients for CCCC B.C.

$$
\begin{aligned}
& K(3,2)=-I_{m p} I_{n q}\left[\alpha_{m} \beta_{n}\left[-4 \frac{g_{4}^{c y x}}{h_{c}^{4}}-8 \frac{g_{5}^{c y x}}{h_{c}^{5}}-16 \frac{g_{6}^{c y x}}{h_{c}^{6}}\right]\right], \\
& K(8,10)=-I_{m p} I_{n q}\left[\alpha_{m} \beta_{n} h_{b}^{2}\left[-\frac{g_{4}^{c y x}}{h_{c}^{4}}+4 \frac{g_{5}^{c y x}}{h_{c}^{5}}-4 \frac{g_{6}^{c y x}}{h_{c}^{6}}\right]\right], \\
& K(13,7)=-I_{m p} I_{n q}\left[\alpha_{m} \beta_{n} h_{t}\left[\frac{g_{2}^{c x z}}{h_{c}^{2}}+2 \frac{g_{3}^{c x z}}{h_{c}^{3}}-4 \frac{g_{4}^{c x z}}{h_{c}^{4}}-8 \frac{g_{5}^{c x z}}{h_{c}^{5}}+\frac{g_{3}^{c x z}}{R_{c y} h_{c}^{2}}+2 \frac{g_{4}^{c x z}}{R_{c y} h_{c}^{3}}-4 \frac{g_{5}^{c x z}}{R_{c y} h_{c}^{4}}-8 \frac{g_{6}^{c x z}}{R_{c y} h_{c}^{5}}\right]\right] .
\end{aligned}
$$

